\title{
Patient engagement in research related to accelerated surgical care and treatment for hip fracture
}

\author{
Michael H. McGillion RN PhD, Lee Lin-Rogano, Flavia Kessler Borges MD PhD
}

Cite as: CMAJ 2018;190(Suppl 1):S38-S39. doi: 10.1503/cmaj.180447

E ach year, millions of adults worldwide experience a hip fracture with devastating consequences. ${ }^{1-3}$ Following hip fracture, the 30-day mortality rate is $9.3 \%$ (95\% confidence interval [Cl] 8.8\%-9.9\%) for men and 5.2\% (95\% Cl 4.9\%-5.4\%) for women. ${ }^{4}$ Those who survive to 30 days after the fracture are at substantial risk of disability. Although preliminary data suggest that early surgical treatment may improve outcomes, research shows that most patients receiving standard care in Ontario will wait about 38 hours for surgery. ${ }^{5}$ Two main factors that delay surgery are preoperative medical clearance and operating room access. In a pilot trial (two centres in Hamilton, Ontario, and one centre in Maharashtra, India), the Hip Fracture Accelerated Surgical Treatment and Care Track (HIP ATTACK) team established that both factors were modifiable at study centres, allowing for rapid access to surgical intervention within six hours of diagnosis. ${ }^{6}$

Our group designed HIP ATTACK, a multicentre (61 centres, 18 countries, 4 continents), international randomized controlled trial (ClinicalTrials.gov NCT02027896), to assess the effect of accelerated surgical care versus standard care on the risk of 90-day all-cause mortality and major perioperative complications in adults with a hip fracture. The study involves 3000 participants who receive either accelerated medical clearance and surgery, or standard care. Those randomly assigned to accelerated care gain medical clearance by a dedicated HIP ATTACK medical specialist, and approval by a staff orthopedic surgeon and anesthesiologist. Medically cleared patients are moved into the next orthopedic elective operating room slot, gaining priority over scheduled elective cases. Included are consenting patients aged 45 years and older with a hip fracture (from a low-energy mechanism) that requires surgery. Patients are followed throughout their hospital stay, and at 30 days, 90 days and 1 year after surgery.

The HIP ATTACK approach to patient engagement is guided by the Canadian Institutes of Health Research Strategy for PatientOriented Research (SPOR) Patient Engagement Framework. ${ }^{7}$ Our current group of 19 patient partners participated in the HIP ATTACK trial (control or intervention arm) and were recommended by trial surgeons. These individuals were screened to ensure they were free of cognitive deficits after surgery, and that

\section{KEY POINTS}

- The Hip Fracture Accelerated Surgical Treatment and Care Track (HIP ATTACK) trial is a multicentre international trial, designed to assess the impact of accelerated surgical care versus standard care on the risk of 90-day all-cause mortality and major perioperative complications in adults with a hip fracture.

- Patient engagement in the study spans research governance, strategy and methodology.

- Fatigue management can be a challenge to patient engagement among the hip fracture population; patient-engagement activities are limited to half-day sessions, with additional time built in for stretch and nutrition breaks to optimize the comfort of patient partners.

- Terms of involvement are expressed in a patient-engagement committee charter to make role expectations and terms of membership clear.

they were capable of travel to our trial methods centre for patient-engagement activities. We also invite family and caregiver involvement. Membership is revisited every six to eight months, and new members are added as needed. Committee representation also includes hospital-site decision-makers and heath-policy experts. Committee members self-select to participate in our activities based on individual interest and availability.

Our patient-engagement techniques span research governance, strategy and methods; key examples include governance auditing, Web-based knowledge dissemination and trialoutcome evaluation. Informed by the Canadian Policy Research Network Health Care Accountability Papers, ${ }^{8}$ which stress social accountability, we designed the role of a governance auditor to enable patient representatives to co- and self-govern. During our patient-engagement activity meetings, governance auditors maintain an audit trail of strategy- and execution-related decisions in order to guide ongoing activities. Based on patient feedback, we now use prepopulated audit forms to allow for ease of documentation. Two committee members volunteer as governance auditors at each meeting, to facilitate cross-validation of 
decisions made. Examples of decisions typically audited include frequency and agenda for upcoming meetings, and ongoing priority setting for patient engagement.

With respect to Web-based knowledge dissemination, we prioritize experiential knowledge by capturing "word on the street" video commentaries from our patient partners, for future incorporation into end-of-grant knowledge dissemination. These brief (40 to $60 \mathrm{~s}$ ) commentaries target personal experience of the intervention. These commentaries will be disseminated through Reducing Global Perioperative Risk, a multimedia knowledge-dissemination resource centre developed in partnership with Elsevier. This resource centre is linked to Elsevier's global online readership, with clinician and public end users in more than 150 countries, across 5 continents. In previous work (based on an earlier prototype), interrupted time series analyses showed that inclusion of "word on the street" videos via social media substantially increased global uptake of the resource centre, including total users, user sessions and page views. ${ }^{9}$

We incorporate outcome evaluation into our patientengagement strategy by reviewing the primary (pertaining to 90-day all-cause mortality, and a composite of perioperative complications) and secondary outcome definitions (pertaining to other complications, quality of life, health system use and cost) of the HIP ATTACK trial with our patient partners. We use an interactive audience-response system so patient partners can tell us, from their perspective, which outcomes matter most. We periodically collect this information with new patient partners to inform our future media and communications strategy, once trial results become available. To date, 16 patient partners have participated.

Although we originally intended that outcome evaluation would solely inform our future media strategy, this activity yielded an unanticipated benefit. Our patient representatives expressed that in addition to trial outcomes, we should also collect qualitative data pertaining to patient experience of the intervention. The decision was made to co-create a trial exit interview according to the principles of Patients First: Action Plan for Health Care, the Ontario Ministry of Health and Long-Term Care blueprint for health care transformation (www.health.gov.on.ca/en/ms/ecfa/healthy_ change). Our patient-engagement committee held a full-day retreat to generate exit interview questions that would solicit patient experiences according to Patients First principles. Committee members voted on and reduced 32 initial interview questions to 12 for inclusion in the final exit interview schedule (three items per Patients First principle), which will be used in an upcoming qualitative substudy of the experience of the HIP ATTACK intervention.

Anecdotal feedback from our patient partners indicates that our patient-engagement strategies are both acceptable and feasible. Despite our successes, a challenge to patient engagement among the hip fracture population has been fatigue management. In the past, we conducted some of our patientengagement activities as full-day events, which proved to be fatiguing. We now limit patient-engagement activities to half-day sessions, and we build in additional time for stretch and nutrition breaks to improve comfort. We also schedule additional time for transition periods from one activity to the next. Finally, based on feedback from our patient partners, we have detailed terms of involvement in a patient-engagement committee charter so that new patient partners have explicit understanding of committee role expectations and terms of membership.

To summarize, the SPOR Patient Engagement Framework ${ }^{7}$ has been a helpful tool for informing our patient-engagement strategy, and has yielded additional benefit in terms of informing an upcoming qualitative substudy on patient experience of the HIP ATTACK intervention. A recent survey of 38 investigators across Canada identified common concerns about patient involvement in research relating to lack of clarity about the meaning and impact of patient engagement. ${ }^{10}$ In applying SPOR principles, we have been able to be creative in terms of devising authentic patient-engagement strategies that are both practical and useful for advancing the HIP ATTACK research agenda.

\section{References}

1. Woolf AD, Pfleger B. Burden of major musculoskeletal conditions. Bull World Health Organ 2003;81:646-56.

2. Leslie WD, O'Donnell S, Jean S, et al.; Osteoporosis Surveillance Expert Working Group. Trends in hip fracture rates in Canada. JAMA 2009;302:883-9.

3. LeBlanc ES, Hillier TA, Pedula KL, et al. Hip fracture and increased short-term but not long-term mortality in healthy older women. Arch Intern Med 2011;171:1831-7.

4. Brauer CA, Coca-Perraillon M, Cutler DM, et al. Incidence and mortality of hip fractures in the United States. JAMA 2009;302:1573-9.

5. Pincus D, Wasserstein D, Ravi B, et al. Reporting and evaluating wait times for urgent hip fracture surgery in Ontario, Canada. CMAJ 2018;190:E702-9.

6. Hip Fracture Accelerated Surgical Treatment and Care Track (HIP ATTACK) Investigators. Accelerated care versus standard care among patients with hip fracture: the HIP ATTACK pilot trial. CMAJ 2014;186:E52-60.

7. Strategy for Patient-Oriented Research (SPOR): putting patients first - Strategy for patient-oriented research: patient engagement framework. Ottawa: Canadian Institutes of Health Research; 2018. Available: www.cihr-irsc.gc.ca/e/documents /spor_framework-en.pdf (accessed 2018 Mar. 22).

8. Abelson J, Gauvin F-P. Engaging citizens: one route to health care accountability. Health Care Accountability Papers no. 2. Ottawa: Canadian Policy Research Networks Inc. (CPRN); 2004. Available: http://citeseerx.ist.psu.edu/viewdoc/ download?doi=10.1.1.539.1539\&rep=rep1\&type=pdf (accessed 2018 Mar. 22).

9. McGillion M, Pilote L, Jolicoeur E, et al. Interim performance metrics of an innovative, web-based Canadian Journal of Cardiology knowledge dissemination platform. Can J Cardiol 2014;30(Suppl.):S315.

10. Carroll SL, Embuldeniya G, Abelson J, et al. Questioning patient engagement: research scientists' perceptions of the challenges of patient engagement in a cardiovascular research network. Patient Prefer Adherence 2017;11:1573-83.

More information on this project is available at www.ossu.ca/ IMPACTAwards.

\section{Competing interests: None declared.}

This article was solicited and has been peer reviewed.

Affiliations: McMaster University (McGillion, Borges); Population Health Research Institute (McGillion, Borges); patient partner (LinRogano), Hamilton, Ont.

Contributors: All authors contributed to the conception and design of the work, drafted the manuscript, revised it critically for important intellectual content, gave final approval of the version to be published and agreed to be accountable for all aspects of the work.

Funding: This work was supported by OSSU (the Ontario SPOR [Strategy for Patient-Oriented Research] SUPPORT [Support for People and Patient-Oriented Research and Trials] Unit).

Acknowledgements: The authors thank the dedicated, engaged patient representatives of the HIP ATTACK trial who have made this work possible.

Correspondence to: Michael McGillion, mmcgill@mcmaster.ca 